\title{
Nouveaux lasers à fibre pour polariser les spins nucléaires d'un gaz d'hélium : applications médicales à l'Imagerie par Résonance Magnétique
}

\author{
M. Leduc et E. Courtade \\ Laboratoire Kastler Brossel, \\ École Normale Supérieure, \\ 24 rue Lhomond, \\ 75231 Paris Cedex 05, France \\ leduc@lkb.ens.fr
}

Résumé. Nous décrivons ici un nouveau dispositif laser fonctionnant dans l'infrarouge proche et donnant plusieurs watts de puissance en continu, qui est utilisé pour polariser nucléairement du gaz d'hélium3 par la méthode du pompage optique indirect via le niveau métastable $2^{3} S_{1}$ de l'atome d'hélium [1]. La source laser est basée sur un amplificateur à fibre double cœur dopée Ytterbium, excité par un laser à fibre de largeur spectrale adaptée au pompage optique. Les résultats pour la polarisation obtenue avec cette source lumineuse sont ici donnés. Une application est présentée : l'Imagerie par Résonance Magnétique (IRM) des poumons utilisant du gaz d'hélium-3 ainsi «hyperpolarisé » et inhalé. Cette méthode permet de visualiser les volumes creux à l'intérieur des poumons et de caractériser leur dynamique de ventilation. Elle pourrait être utilisée pour étudier la fonctionnalité du poumon et pour le diagnostic précoce de certaines pathologies telles que l'obstruction chronique des voies respiratoires ou l'emphysème. 


\section{INTRODUCTION}

Dès que la possibilité de polariser nucléairement du gaz d'hélium-3 par la méthode du pompage optique indirect a été découverte, des applications ont été développées dans de nombreux domaines, de la physique statistique et de la physique nucléaire jusqu'à la médecine $[2,3]$. Une méthode consiste à combiner le pompage optique de l'atome d'hélium dans l'état métastable $2^{3} \mathrm{~S}_{1}$ avec les collisions d'échange de métastabilité [1]. Initialement le pompage optique s'effectuait avec des lampes à décharge et les premières possibilités d'utilisation du gaz polarisé en magnétométrie par Résonance Magnétique Nucléaire (RMN) sont apparues. D'autres développements ont pu vraiment commencer lorsque les premiers lasers ont été mis au point pour la longueur d'onde dans l'infrarouge proche correspondant à la raie intense $2^{3} \mathrm{~S}_{1}-2^{3} \mathrm{P}$ à 1083 nm utilisée pour le pompage optique de l'hélium. Ce furent d'abord des lasers à centres colorés (centres $\mathrm{F}_{2+}$ dans des cristaux de $\mathrm{NaF}$ ) [4], dont l'émission présentait l'inconvénient de n'être pas stable dans le temps. Ils furent ensuite remplacés par des lasers à cristaux dopés de Néodyme (LNA) : pompés par des lasers à gaz, ces cristaux fournissent une puissance de l'ordre de $100 \mathrm{~mW}$ [5] ; ils peuvent aussi être pompés par des diodes lasers, ce qui donne des sources compactes pour la magnétométrie; la version dans laquelle de grands barreaux cristallins sont excités par des lampes à flash donne des puissances importantes, de l'ordre de plusieurs watts [6]. Plus récemment des diodes lasers à 1083 nm (provenance SDL) sont apparues sur le marché avec une puissance maximale de $50 \mathrm{~mW}$ [7]. Enfin, depuis 1998, des amplificateurs à base de fibre dopée Ytterbium (Yb) sont commercialisés par IR Polus [8] et OptoCom. La combinaison d'une diode laser et d'un tel amplificateur donne une source dite MOPFA délivrant une puissance continue de l'ordre du watt. OptoCom vient de développer une source laser où la diode laser est remplacée par un oscillateur lui aussi à fibre dopée $\mathrm{Yb}$. Cette nouvelle génération de sources lasers à $1083 \mathrm{~nm}$, décrite au paragraphe 3, est maintenant utilisée par notre équipe à l'ENS pour les applications à $1^{\prime} I R M$.

Ces avancées dans le domaine des lasers ont permis d'abord d'élargir le champ des fluides quantiques aux propriétés du gaz et du liquide d'hélium-3 polarisé à basse température [9]. Elles ont aussi profité au domaine des cibles polarisées, permettant d'arriver à des résultats fondamentaux sur la structure du neutron [10] et fournissant une nouvelle génération de polariseurs large bande pour les faisceaux de neutrons [11]. L'application la plus récente concerne un domaine inattendu : l'IRM avec du gaz d'hélium polarisé nucléairement par laser et inhalé par un patient. Cette nouvelle méthode d'imagerie a été découverte en 1994 avec une expérience portant sur des animaux et utilisant du xénon polarisé [12]. Elle est aujourd'hui considérée comme un outil prometteur en médecine pulmonaire car elle permet une exploration à la fois de l'anatomie et de la fonction des voies respiratoires chez l'homme.

Cet article comprend un rappel sur le pompage optique de l'hélium et détaille les nouveaux lasers à fibres utilisés depuis peu pour les explorations en IRM, puis les résultats de polarisation obtenus avec ces sources. La méthode de l'IRM avec gaz polarisé est ensuite présentée ainsi que ses potentialités médicales. 


\section{POMPAGE OPTIQUE DE L'HÉLIUM-3}

L'atome d'hélium-3 est polarisé nucléairement par pompage optique. Deux méthodes différentes, toutes deux faisant appel au pompage optique, se sont développées en parallèle et bénéficient aujourd'hui des progrès sur les sources lasers.

L'atome d'hélium dans son état fondamental $1^{1} S_{0}$ n'est pas propice au pompage optique direct, en particulier parce que les raies de résonance optique qui le relient aux niveaux excités tombent dans le domaine de l'ultra violet. La méthode d'orientation combine le pompage optique d'une autre espèce atomique et un transfert de polarisation par des processus collisionnels. Dans un cas c'est un atome alcalin, le plus souvent du rubidium, qui est polarisé par pompage optique à $795 \mathrm{~nm}$ avec des réseaux de diodes lasers de puissance (plusieurs dizaines voire centaines de watts); dans l'autre c'est l'atome d'hélium, porté dans l'état excité $2^{3} \mathrm{~S}_{1}$ métastable par une décharge radiofréquence, qui est polarisé à l'aide de la raie intense $2^{3} \mathrm{~S}_{1}-2^{3} \mathrm{P}$ à $1083 \mathrm{~nm}$. La première méthode est fondée sur les collisions dites d'échange de spin entre atomes de rubidium et d'hélium [13], la seconde utilise les collisions d'échange de métastabilité entre atomes d'hélium-3 dans les états $2^{3} S_{1}$ et $1^{1} S_{0}[14]$.

La voie par échange de spin avec un alcalin permet de polariser des volumes limités (par exemple $200 \mathrm{~cm}^{3}$ ) dans une cellule à forte pression (plusieurs atmosphères) en un temps de l'ordre de plusieurs heures [15]. L'échange de métastabilité est une méthode qui ne permet de polariser le gaz qu'à des pressions plus faibles, de l'ordre $\mathrm{du}$ mbar, mais avec des temps de construction de la polarisation beaucoup plus courts, de l'ordre de quelques secondes; l'efficacité de ce processus de pompage est plus grande, environ 2 photons seulement sont nécessaires pour polariser un noyau. Les taux de polarisation atteints sont sensiblement supérieurs, les volumes polarisés peuvent atteindre plusieurs litres et, d'une façon générale, les taux de production du gaz polarisé sont nettement plus élevés.

La voie du "tout hélium », utilisant des lasers à $1083 \mathrm{~nm}$, est celle qui a été choisie par notre équipe à l'ENS et par les groupes de Mayence avec lesquels nous collaborons actuellement, pour les applications à l'imagerie médicale. Les raisons de ce choix résultent de la possibilité de polariser mieux et plus rapidement de plus grandes quantités de gaz. C'est la puissance lumineuse à $1083 \mathrm{~nm}$ qui limite le taux de production maximum de l'hélium polarisé par la méthode du pompage optique par échange de métastabilité. Les lasers LNA pompés par lampes sont pour l'instant les sources les plus puissantes disponibles. Ils pourraient être remplacés par les lasers à fibres dopées $\mathrm{Yb}$, susceptibles de délivrer des puissances de plusieurs watts.

\section{LE LASER À FIBRE DOPÉE Yb}

Nous décrivons ici une source entièrement constituée de fibres optiques et développée par la société OptoCom Innovation [16]. Il s'agit d'un appareil compact et robuste ne nécessitant pas de réajustement de la part de l'utilisateur, particulièrement bien adapté aux applications en milieu hospitalier. Le schéma de cette source est représenté sur la partie gauche de la figure 1. Elle consiste en un oscillateur maître de 


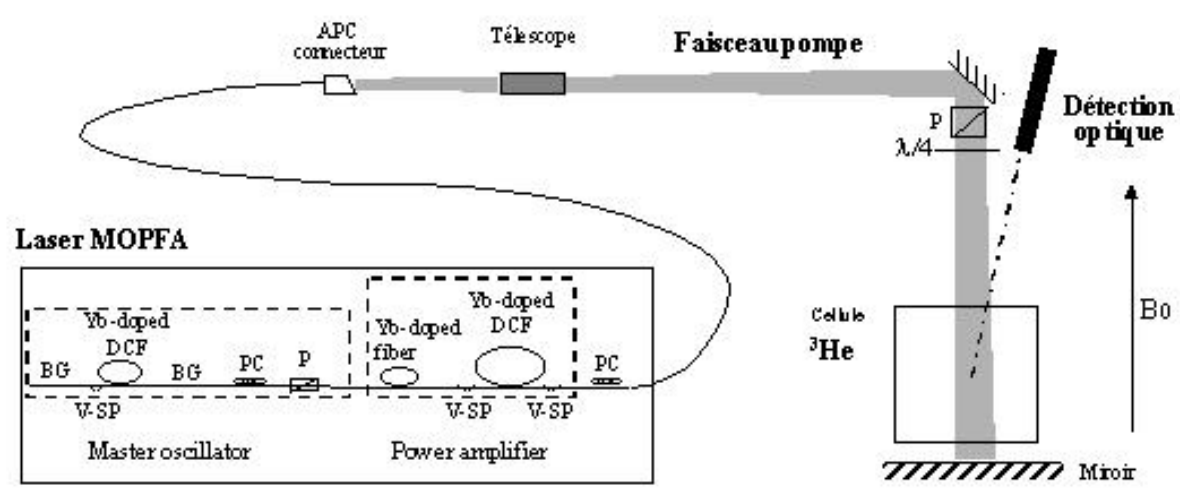

FIg. 1. - Montage expérimental utilisé pour mesurer les taux de pompage optique dans un gaz d'hélium-3. La gauche de la figure représente les éléments de la source laser OptoCom comportant un oscillateur maître et un ampli de puissance, tous deux constitués de fibres dopées Ytterbium (voir texte).

faible puissance («master oscillator») et un amplificateur de fort gain ("power amplifier »). Ce système diffère de celui de la référence [8], utilisé dans les expériences de refroidissement de l'hélium-4 métastable par laser, où le même type d'amplificateur est excité par une diode laser affinée spectralement. Notons que dans tous les cas, la puissance du laser maître nécessaire à l'excitation de l'amplificateur est faible, de l'ordre du milliwatt.

Le laser maître présenté ici (Fig. 1) est constitué d'une fibre dopée Yb à double cœur (DCF), placée dans une cavité Fabry-Pérot comprenant un réseau de Bragg (BG) à chaque extrémité en guise de réflecteurs. Sa largeur spectrale d'environ $1,8 \mathrm{GHz}$ FWHM est bien adaptée au profil Doppler d'absorption des atomes d'hélium métastable à pomper ( $2 \mathrm{GHz}$ à $300 \mathrm{~K})$. Le balayage en fréquence du laser s'effectue en changeant la température des réseaux de Bragg. Dans une version toute récente, on agit plus directement sur la longueur de la cavité par une céramique piezo-électrique. En fonctionnement normal, un contrôle de la température dans la boîte du laser assure une bonne stabilité de la fréquence et de l'intensité de la lumière émise.

L'amplificateur comporte deux parties : un «booster » composé d'une fibre à double cœur d'environ $10 \mathrm{~m}$ de long et un ampli préalable, également à fibre dopée $\mathrm{Yb}$, mais pas à double cœur. L'oscillateur maître et le «booster » sont tous deux pompés longitudinalement par des réseaux de diodes lasers à $970 \mathrm{~nm}$ couplées longitudinalement dans la fibre (V-SP : V-groove side pumped). Par contre, le préamplificateur à fibre situé entre eux est pompé par la lumière d'émission spontanée émise par le «booster » et contribue au gain total de l'étage d'amplification. 
Le contrôle de la polarisation lumineuse à la sortie du laser maître s'effectue à l'aide d'un contrôleur de polarisation (PC) et d'un polariseur $(\mathrm{P})$ : les réglages sont faits par le constructeur et l'utilisateur n'y a pas accès. Par contre, un polariseur en sortie de l'amplification permet à l'utilisateur d'adapter la polarisation à celle requise pour son expérience. Les isolateurs optiques intérieurs à la source laser ne figurent pas sur la figure 1. Notons qu'un isolateur situé à la sortie empêche le retour de lumière réfléchie par l'expérience susceptible de perturber le fonctionnement de l'oscillateur. De même le connecteur APC empêche l'amplificateur d'osciller.

La source ici décrite délivre une puissance continue de 2 watts. Il peut exister des versions plus puissantes du même laser ( 5 watts chez OptoCom) : le laser maître est le même mais l'étage d'amplification est plus long. La firme IR Polus commercialise également un amplificateur du même type donnant 7 watts de puissance de sortie.

\section{RÉSULTATS POUR LA POLARISATION NUCLÉAIRE}

Le pompage optique utilise le faisceau selon le schéma de la figure 1 (partie droite). Le faisceau est étendu par un télescope jusqu'à un diamètre de l'ordre de $2 \mathrm{~cm}$. Il traverse la cellule à polariser et est renvoyé sur lui même par un miroir pour augmenter l'efficacité du pompage. Avant d'entrer dans la cellule, il est très soigneusement polarisé circulairement par une cube polariseur $(\mathrm{P})$ suivi d'une lame quart d'onde $(\lambda / 4)$ (moins de $1 \%$ de mauvaise polarisation). Le faisceau de pompage est colinéaire à un champ magnétique statique $B_{0}$ de quelques milliTesla. La polarisation des noyaux de l'hélium est mesurée par une méthode optique décrite dans la référence [17], qui consiste à mesurer le taux de polarisation circulaire de la raie de l'hélium à $668 \mathrm{~nm}$ émise par la décharge.

La figure 2 montre l'enregistrement de la construction de la polarisation nucléaire dans une cellule contenant du gaz d'hélium-3 à une pression de 1.3 mbar. Le laser utilisé est celui décrit précédemment : la puissance lumineuse incidente sur la cellule est de 1,40 watt. La polarisation nucléaire $M$ atteint son niveau d'équilibre voisin de $70 \%$ presque exponentiellement, avec une constante de temps $T_{p}$ de l'ordre de $7 \mathrm{~s}$. Les valeurs de $M$ et $T_{p}$ dépendent des paramètres du laser d'une façon qui est analysée dans la référence [18].

Les applications médicales nécessitent une production de gaz très polarisé et dense (i.e. à pression atmosphérique pour pouvoir être inhalé). Comme on l'a vu au paragraphe 2, la méthode de pompage optique par échange de métastabilité permet d'avoir de grandes polarisations mais à de faibles pressions (de l'ordre du mbar). Un dispositif de compression à pistons de titane a été mis au point à Mayence. Il permet de monter la pression jusqu'à plusieurs atmosphères avec une faible dépolarisation du gaz. Avec une puissance de laser de $16 \mathrm{~W}$, obtenue avec des lasers LNA pompés par lampes [11], on produit ainsi 0,6 bar-litre par heure avec une polarisation $M$ de $50 \%$. Des versions moins sophistiquées de compresseur sont actuellement développées pour les besoins de l'imagerie médicale [19] : on ne cherche plus nécessairement à obtenir la pression finale et la polarisation les plus grandes possibles, par contre, le taux de production est un paramètre que l'on souhaite optimiser dans la perspective d'examens médicaux en série. Le but poursuivi est alors la construction d'un polariseur de gaz utilisable de façon commode in situ en milieu hospitalier. 


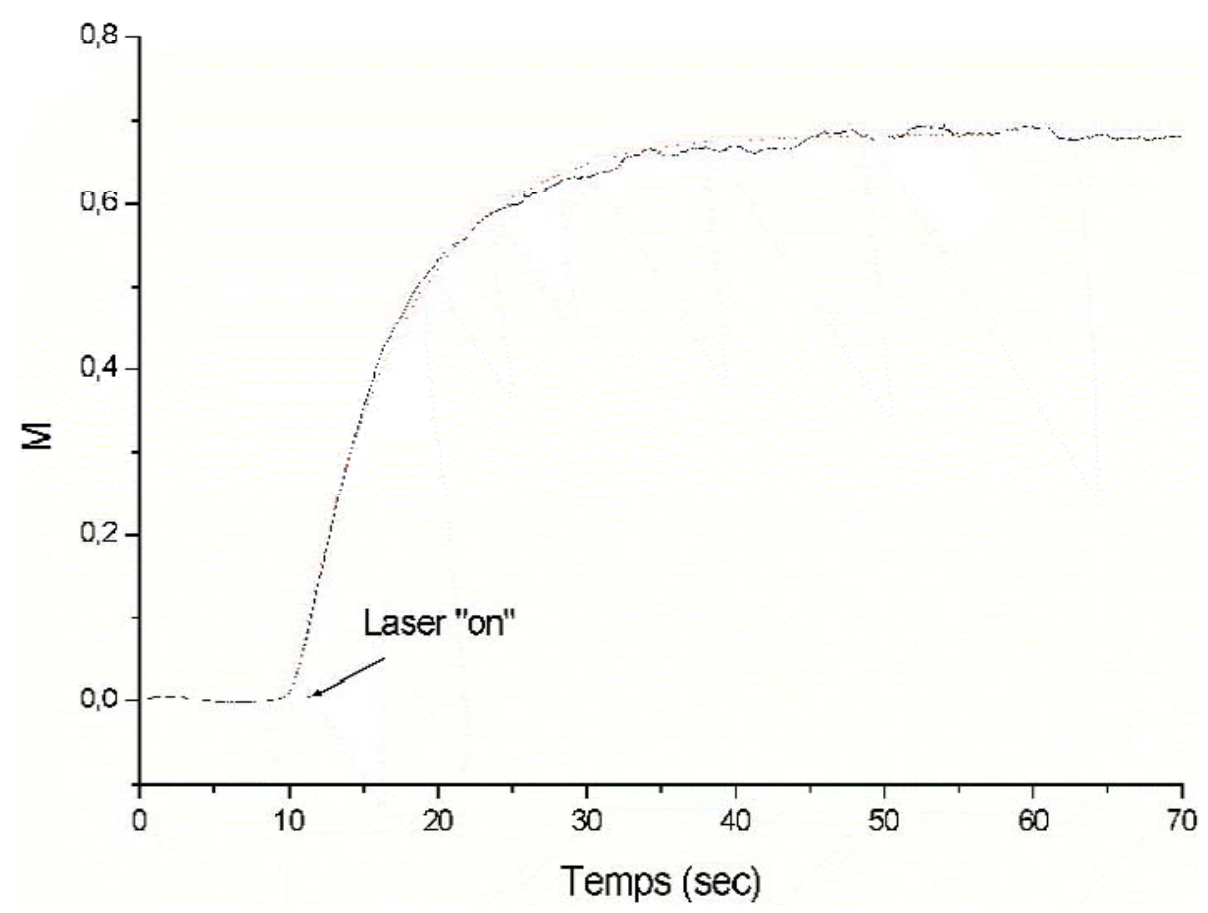

FIG. 2. - Construction de la polarisation nucléaire $M$. Le laser à fibre OptoCom donne une puissance incidente de 1,4 watt sur une cellule cylindrique $(5 \mathrm{~cm}$ de diamètre et $5 \mathrm{~cm}$ de haut) contenant de l'hélium-3 gazeux à 1,33 mbar. La fréquence du laser est accordée sur la transition $\left(2^{3} \mathrm{~S}_{1}, F=3 / 2-2^{3} \mathrm{P}_{0}, F=1 / 2\right)$. Ce signal de détection optique est acquis avec une constante de temps de $1 \mathrm{~s}$. En pointillés est représenté un fit exponentiel du signal qui donne une polarisation d'équilibre $M$ de $68,5 \%$ et un temps de pompage $T_{p}$ de $7 \mathrm{~s}$.

\section{L'IMAGERIE PAR RÉSONANCE MAGNÉTIQUE AVEC DU GAZ POLARISÉ}

L'IRM avec gaz polarisé [21] diffère de l'IRM conventionnelle en ce que l'on observe la résonance des noyaux du gaz rare inhalé et non ceux des protons. La fréquence de résonance est accordée sur celle du noyau d'hélium (33 MHz par Tesla) et non plus celle des protons (42 MHz par Tesla). En IRM conventionnelle on fait l'image des tissus, des organes, des os ou du sang. Par contre, en IRM avec hélium polarisé, on observe les volumes creux remplis par le gaz inhalé. Une autre différence est que la polarisation des protons résulte de l'immersion du patient dans un champ magnétique élevé, alors que celle du gaz inhalé est produite antérieurement à l'examen et par des techniques laser à l'extérieur du corps. La densité des noyaux d'hélium inhalé est beaucoup plus faible que celle des protons dans les tissus, mais pour ce 
qui concerne l'intensité des signaux RMN, ceci est compensé par la valeur de la polarisation nucléaire qui est de 4 ou 5 ordres de grandeur plus élevée.

La différence principale de l'IRM avec hélium avec l'IRM conventionnelle est que la polarisation des noyaux est hors d'équilibre et donc métastable; elle ne se reconstitue pas au cours de l'examen. Un paramètre important est le temps de relaxation longitudinal $T_{1}$ de l'aimantation. Les valeurs de $T_{1}$ sont de l'ordre de plusieurs heures pour le gaz conservé à l'abri des gradients de champ magnétique dans des cellules de verre [22]. Elles tombent à 8 ou $10 \mathrm{~s}$ dans les poumons à cause de la présence d'oxygène paramagnétique dans le gaz [23]. Le temps de relaxation transversale $T_{2}^{*}$, qui caractérise l'amortissement des signaux de précession RMN, est de l'ordre de quelques millisecondes pour les protons mais peut être très long (plusieurs secondes) dans le cas de l'hélium inhalé [24]. Toutefois, il est en général raccourci par les inhomogénéités de champ magnétique, dont certaines sont liées à la différence de susceptibilité magnétique entre le gaz et les tissus du poumon.

Une autre caractéristique de l'IRM avec l'hélium est que le gaz diffuse beaucoup plus rapidement dans les espaces creux que les protons dans l'eau. La diffusion des atomes lorsque les gradients de l'imagerie sont appliqués entraîne une perte de signal. La résolution des images IRM obtenues avec l'hélium se trouve ainsi limitée : la définition est au mieux de l'ordre du millimètre. Inversement, l'IRM permet une mesure du coefficient apparent de diffusion du gaz dans la géométrie confinée constituée par les bronches et les alvéoles du poumon. Ce coefficient donne une indication sur la distribution moyenne des tailles des voies aériennes pulmonaires et pourrait être un moyen de diagnostic de certaines pathologies comme l'obstruction chronique des bronches, l'emphysème ou l'asthme [25].

La stratégie des recherches en IRM avec l'hélium polarisé s'oriente aujourd'hui vers l'adaptation des séquences RMN aux particularités de cette méthode d'imagerie : acquisition rapide des images [23], mesure de la diffusion du gaz, étude des échanges d'oxygène par l'intermédiaire des temps de relaxation, etc. Les champs magnétiques importants (quelques Teslas) utilisés dans les tomographes traditionnels pour le proton permettent d'augmenter la polarisation de ce dernier et donc de conditionner le rapport signal/bruit. Avec l'hélium-3 polarisé, l'aimantation du gaz est indépendante du champ appliqué et le signal/bruit est constant si à la fois le signal et le bruit augmentent linéairement avec le champ. Nous travaillons avec 1'U2R2M [29] à bas champ (0,1 Tesla) avec l'hélium-3 gazeux polarisé pour ces raisons. Dans la perspective d'une production industrielle, le bas champ est intéressant car il peut être créé à partir d'aimants résistifs classiques, alors qu'il faut mettre en oeuvre pour des champs de quelques Teslas des technologies beaucoup plus coûteuses (aimants supraconducteurs refroidis à l'hélium liquide).

La figure 3 montre une image des poumons d'un volontaire sain obtenue par IRM avec de 1'hélium-3 polarisé à 15\% environ. La quantité d'hélium-3 utilisée ici est modeste $\left(30 \mathrm{~cm}^{3}\right.$.atm) et le gaz est mélangé à un autre gaz neutre juste avant inhalation. La résolution spatiale de l'image en 2D est de l'ordre de $6 \mathrm{~mm}$. L'utilisation de plus grandes quantités de gaz mieux polarisées permet d'obtenir des images en 3D et de plus grande définition. 


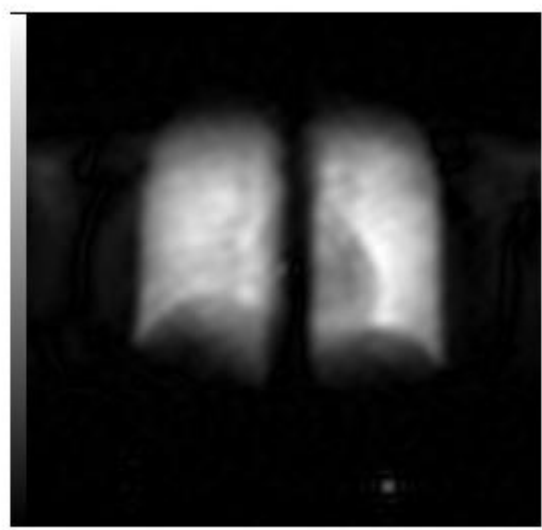

Projection frontale coronal

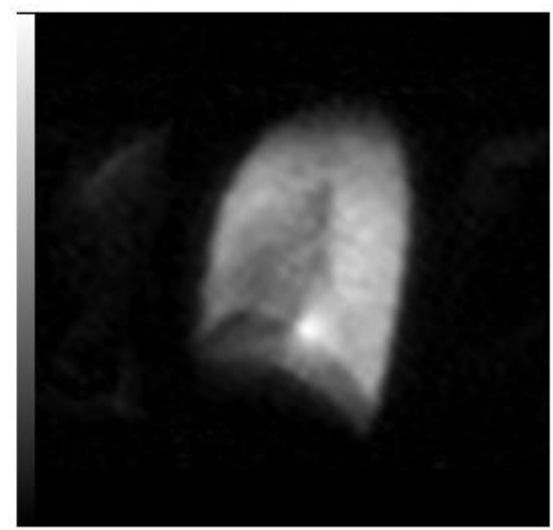

Projection sagittale sagittal

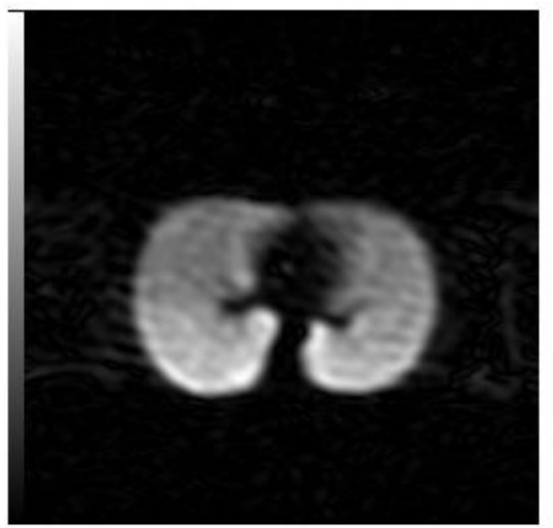

Coupe transversale axial slice
U2R2M-Orsay

LKB - Paris

FIG. 3. - Images de poumons humains en champ faible (0,1 Tesla) en IRM d'hélium-3 polarisé pour un temps d'acquisition de $0,3 \mathrm{~s}$ avec une séquence multi-échos de spin. Ces images ont été obtenues avec des quantités modestes $\left(30 \mathrm{~cm}^{3}\right.$.atm) d'hélium-3 polarisé à $15 \%$ dilué dans un gaz d'hélium-4 pour pouvoir être inhalé. Ces projections et cette coupe de $5 \mathrm{~cm}$ d'épaisseur montrent la distribution du gaz dans les poumons avec une résolution spatiale d'environ $6 \mathrm{~mm}$ dans les deux directions de l'image.

La méthode de l'IRM avec hélium-3 hyperpolarisé est actuellement développée par plusieurs équipes aux États-Unis et en Europe (France, Allemagne, Angleterre) associant physiciens, radiologues et médecins. Elle donne des informations non seulement sur l'anatomie des poumons mais aussi sur leur ventilation et leur fonctionnalité. Elle entre actuellement dans une phase d'expérimentation clinique sur des 
patients humains et aussi sur des modèles animaux. Les perspectives d'applications médicales au diagnostic des pathologies pulmonaires sont très prometteuses [26,27]. Toutefois le passage de la méthode au stade du développement industriel et de l'utilisation dans les hôpitaux dépendra de nombreux facteurs : le plus important sera sans doute la démonstration pour les médecins que la nouvelle technique, considérée actuellement comme sophistiquée, permet un diagnostic de pathologies plus précoce et plus précis que les méthodes usuelles, et ceci particulièrement s'il existe pour les pathologies en question des traitements appropriés permettant de sauver des vies humaines.

\section{Références}

[1] F.D. Colegrove et al., Phys. Rev. 132 (1963) 2561.

[2] M. Leduc, J. Phys. Colloq. 51 (1990) C6-317.

[3] J. Becker et al., Nucl. Instr. Meth. A 402 (1998) 327.

[4] M. Leduc et al., Rev. Phys. Appl. 17 (1982) 355.

[5] J.M. Daniels et al., JOSAB 4 (1987) 1133.

[6] C.G. Aminoff et al., Rev. Phys. Appl. 24 (1989) 827.

[7] J.S. Major et al., Electron. Lett. 29 (1993) 2121.

[8] S.V. Chernikov et al., Electron. Lett. 33 (1997) 787.

[9] C. Lhuillier and M. Leduc, Ann. Phys. Fr. 10 (1985) 859.

[10] J. Becker et al., Eur. Phys. J A. 6 (1999) 329.

[11] R. Surkau et al., Nucl. Instr. Meth. A 384 (1997) 444.

[12] M. Alberts et al., Nat. 370 (1994) 1999.

[13] T.C. Walker and W. Happer, Rev. Mod. Phys. 69 (1997) 629.

[14] P.J. Nacher and M. Leduc, J. Phys. Paris 46 (1985) 2057.

[15] K.C. Hasson, European Radiology 9 (1999) B16.

[16] S. Bordais and M. Le Flö̈, International Workshop on "hyperpolarized gases in MR" (Les Houches, France, 1999).

[17] M. Pinard and J. van der Linde, Can. J. Phys. 52 (1974) 1615.

[18] M. Leduc et al., Hyperfine Interactions 127 (2000) 443.

[19] P.J. Nacher et al., European Radiology 9 (1999) B18.

[20] T. Gentile et al., European Radiology 9 (1999) B17.

[21] G. Tastevin, Physica Scripta, T86 (2000) 46.

[22] W. Heil et al., Phys. Lett. A 201 (1995) 337.

[23] B. Saam et al., Proc. $7^{\text {th }}$ ISMRM Meeting (1999), ISSN 1065-9888, p. 449.

[24] L. Darrasse et al., C. R. Acad. Sc. Paris 324 (1997) 691.

[25] J.R. Brookeman et al., European Radiology 9 (1999) B21.

[26] H.U. Kauczor et al., J. Magn. Res. Imag. 7 (1997) 53.

[27] E.E. de Lange et al., Radiology 210 (1999) 851.

[28] N.P. Bigelow, P.J. Nacher and M. Leduc, J. Phys. II France 2 (1992) 2159.

[29] L. Darrasse, E. Durand and G. Guillot (U2R2M, Orsay, France). 